\title{
Report on the ASTERICS projects CORELib and ROAst
}

\author{
Cristiano Bozza ${ }^{1,3}$, Carmelo Pellegrino ${ }^{2}$, Simona Maria Stellacci ${ }^{1}$, Bernardino Spisso ${ }^{1, *}$ \\ ${ }^{1}$ INFN Gruppo Collegato di Salerno, Via Giovanni Paolo II, 132, 84084 Fisciano, Italy \\ 2 INFN-CNAF Viale Carlo Berti-Pichat 6/2, 40127 Bologna, Italy \\ ${ }^{3}$ Dipartimento di Fisica Università di Salerno, Via Giovanni Paolo II, 132, 84084 Fisciano, Italy
}

\begin{abstract}
Two projects included in the OBELICS work package of the ASTERICS cluster will be described: CORELib and ROAst. CORELib is a collection of simulated showers induced by primary cosmic rays and a common framework to produce and manage such large production. CORELib provides high-statistics simulation for various astroparticle and astronomy experiments. The simulations are obtained by employing the widely used CORSIKA simulation framework. The parameters of simulations are available and easily accessible to the users. The goal of ROAst (ROot extension for Astronomy) is to extend the ROOT capabilities adding packages and tools for astrophysical research "bridging" the gap between particle physics and astronomy. The focus is on astronomical catalogues integration and on the support for astronomical coordinate transformations, manipulations as well as the graphical representation of astronomical regions of the sky.
\end{abstract}

\section{Introduction}

ASTERICS aims at addressing the synergies and common challenges shared by various Astronomy ESFRI facilities (SKA, CTA, KM3NeT and ELT). It brings together the communities of particle astrophysics, astronomy and astrophysics, as well as other related research infrastructures. The major tasks of ASTERICS are to support and accelerate the implementation of the ESFRI telescopes, to enhance their performance beyond the current state-of-the-art, and to enable interoperation as an integrated, multi-wavelength and multimessenger facility. The ASTERICS task 3.4 (Data ANAlysis/interpretation, D-ANA) is focused on two main themes: tools to analyse and interpret astronomical/astroparticle observations in an efficient manner and tools for accessing to these observations in an appropriate way, allowing efficient remote and distributed analysis. In this report two projects are presented that are being developed, namely CORELib and ROAst.

\footnotetext{
*Corresponding author: spisso@na.infn.it
} 


\section{CORELib: COsmic Ray Event Library}

CORELib is a collection of simulated showers induced by primary cosmic rays and a common framework to produce and manage such large production. Because cosmic rays are a background source for many astroparticle and astronomy experiments, CORELib is designed to satisfy a broad range of needs. The simulations are obtained employing the widely used CORSIKA simulation framework and the GRID infrastructure as the main computational resource. Initially developed to run simulation for KASCADE experiment, CORSIKA (Cosmic Ray Simulation for KAscade) is a detailed Monte Carlo program to follow step by step the evolution of extensive air showers (EAS) produced by the interaction of cosmic rays with our atmosphere. All secondary particles are tracked along their trajectories and their parameters are stored in files when a predefined observation surface is reached. A first production was launched to profile the computational and storage load. The version of CORSIKA used was 7.5000. The GRID computing infrastructure and the KM3NeT Virtual Organisation have been used. The production has been made in order to fill 7 energy ranges of the primary proton:

\begin{tabular}{|l|l|}
\hline Energy range $(\mathrm{GeV})$ & \multicolumn{1}{|c|}{ Number of events } \\
\hline $200-1000$ & 10000000 \\
\hline $10^{3}-10^{4}$ & 10000008 \\
\hline $10^{4}-10^{5}$ & 1000002 \\
\hline $10^{5}-10^{6}$ & 100000 \\
\hline $10^{6}-10^{7}$ & 10000 \\
\hline $10^{7}-10^{8}$ & 1000 \\
\hline $10^{8}-10^{9}$ & 100 \\
\hline
\end{tabular}

Table 1: Number of simulated events for each energy range in the first production.

In the current productions, the zenith angle of primary cosmic rays is between 0 and 89 degrees, the observation level is placed at sea level, while the Standard European Atmospheric Model is used. The input simulation parameters are available and easily accessible to the users, encoded in CORSIKA data-card format. The current production is composed by two samples characterized by different primary energy spectra and different total number of simulated events. The first has spectral index -2 and is composed by 21 Mevents, while the second has a flat spectrum with 10 Mevents. For each of the two samples, four different high-energy hadronic interaction models are evaluated: QGSJET01 with CHARM option, QGSJET01 with TAULEP option, QGSJETII with TAULEP option and EPOS with TAULEP option. A common low-energy model has been chosen: GHEISHA. For each model, the production has been split in 6 energy bins, equally logarithmically spaced from $1 \mathrm{TeV}$ to $10^{9} \mathrm{GeV}$, plus a low-energy bin from 200 to 1000 $\mathrm{GeV}$. Protons and heavier nuclei $(\mathrm{He}, \mathrm{C}, \mathrm{N}, \mathrm{O}, \mathrm{Fe})$-induced showers are simulated. For proton induced showers, two productions are available: with and without taking into account and storing Cherenkov radiation. All the information about every single shower is stored in a SQLite database that can be queried by the user. In order to simplify the access to the library, a dedicate tool named CORANT is used to translate the CORSIKA output files from binary to ASCII. The information about showers is placed in separated files, one for each particle class (Electromagnetic, Hadrons, Muons, and Neutrinos). The whole production of CORELib will soon be available for download through open access at CNAF, the Information Technology national centre of INFN (Italian Institute for Nuclear Physics). 


\section{ROAst: ROot extension for Astronomy}

The ROOT analysis framework is one of the most used software toolsets for particle physics analysis and it is considered the de facto standard for high-energy physics. The goal of ROAst (ROot extension for Astronomy) is to "bridge" the gap with the world of astrophysical research by extending the ROOT capabilities adding packages and tools. The focus is on the integration of astronomical catalogues and on the support for astronomical coordinate transformations, manipulations as well as the graphical representation of regions of the sky. In order to speed-up future extensions to the catalogue system, the general architecture relies on an intermediate abstraction layer and all the catalogue implementations share the same methods simplifying the access. The support for two local catalogues, namely UCAC4 (U.S. Observatory CCD Astrograph Catalog 4) and URAT1, is available; ROAst supports the on-line Virtual Observatory (VO) catalogues and the VizieR on-line catalogue repository. Moreover, ROAst features a set of coordinate and time conversion methods, which are catalogue-independent. The supported astronomical coordinate systems are: equatorial, galactic, ecliptic, horizontal (using Lat-Long or UTM as geographical coordinates) supplied with methods for mutual transformations. ROAst provides a graphic support library which relies on the ROOT graphic tools. A basic set of graphical tools is provided that is fully consistent with the ROOT environment and all ROOT graphical options are automatically integrated. The customization of the graphs is left to the user. Astronomical objects can be extracted by restricting the catalogue to geometrical regions of three different types and using different coordinate system, as shown in Figure 1.
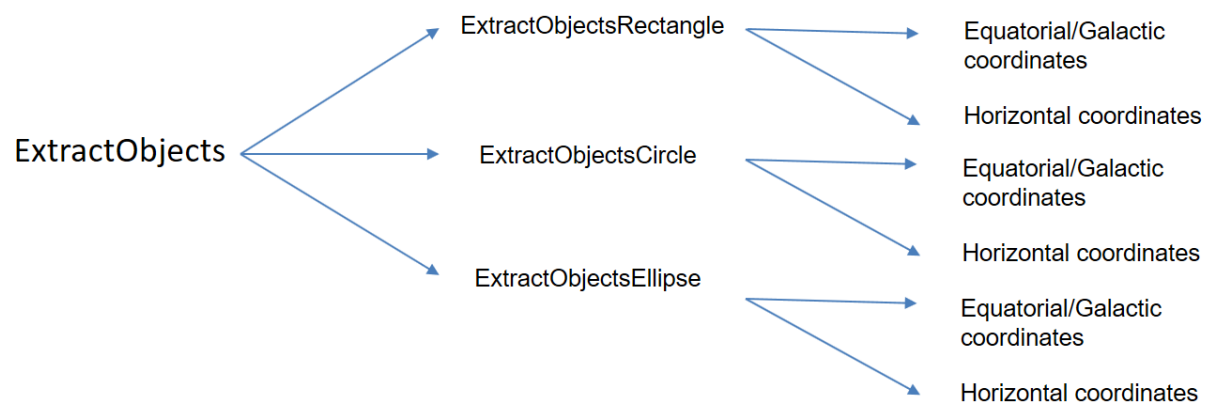

Equatorial/Galactic coordinates

Horizontal coordinates

Figure 1: Scheme of the extraction methods.

The extracted astronomical objects are stored in the public vector ObjectsCollection and can be displayed using the Print method. The main graphical methods are: Draw/DrawFeature which draws the astronomical objects positions, DrawAitoff which can be used to represent the astronomical objects using the equatorial Aitoff projection and DrawSkyMap for the whole sky-map using equatorial Aitoff. The Draw and DrawFeature methods support equatorial, galactic and horizontal astronomical coordinate systems. A decreasing RA axis in the graphs can be enabled by setting to true a global variable.

Finally, ROAst features Moon and Sun position implementing the ELP-2000-82 (Moon) and VSOP-87 (Sun) algorithms for the calculation. 


\subsection{ROAst example}

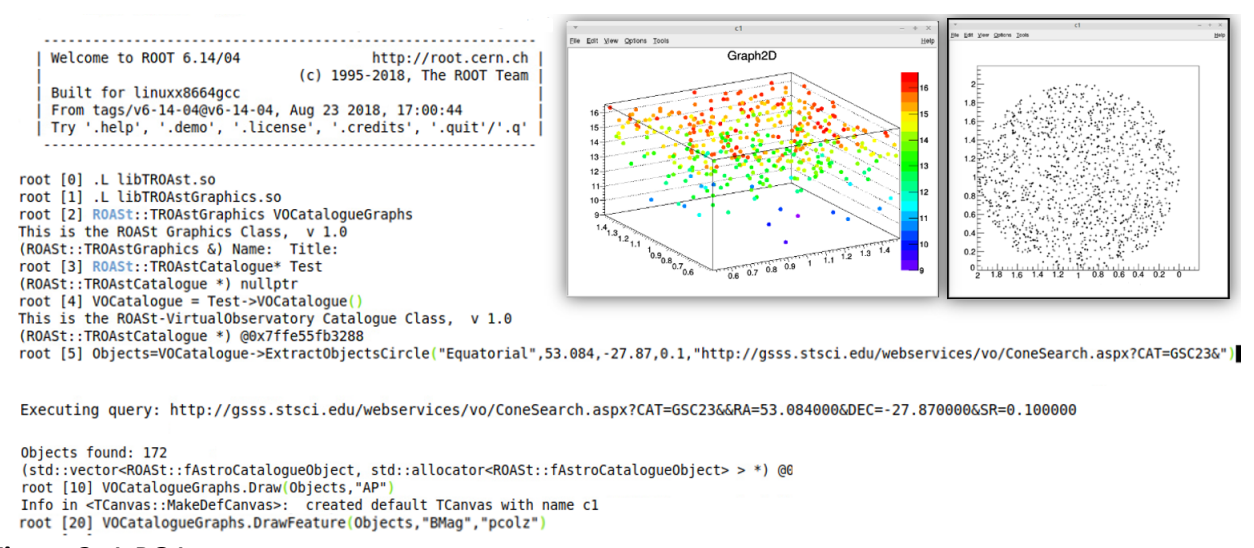

Figure 2: A ROAst use-case.

Fig. 2 shows a use-case in which the user extracts a circular region from an on-line Virtual Observatory via the VOCatalogue::ExtractObjectCircle method. After the extraction, the space region is plotted using the Draw method and the second magnitude is plotted using the DrawFeature method. A C++11-compliant compiler is required, as well as ROOT 6 and the libcurl library, in order to use ROAst. A limited support is also available for ROOT 5 , i.e. without dictionaries.

\section{Conclusions and perspectives}

CORELib uses four different HE-models to provide simulations delivered with a relatively simple ASCII data format for immediate usage. The productions will be accessible through a public server. CORELib provides the full set of parameters used in the simulations, in order to allow more collaborations or institutions to add datasets with/without overlaps. A new production at different observation levels $(2200 \mathrm{~m}, 4000 \mathrm{~m}$ and more) is foreseen jointly with other collaborations (within cooperation agreements to be defined). An updated version of ROAst, with an improved set of graphical tools and fully tested coordinate transformation support, is ready and can be found at https://gitlab.com/Spisso/ASTERICS-ROAst. Next planned developments of ROAst are: improving the class for the coordinate manipulations, adding methods for the positions of the major planets, adding support for image catalogues and allowing asynchronous on-line query (TAP calls).

\section{References}

1. ASTERICS 2020 site https://www.asterics2020.eu/

2. CORSIKA: A Monte Carlo Code to Simulate Extensive Air Showers, D. Heck, J. Knapp, J.N. Capdevielle, G. Schatz, T. Thouw, Forschungszentrum Karlsruhe Report FZKA 6019 (1998)

3. Letter of Intent for KM3NeT 2.0, The KM3NET Collaboration, J.Phys. G 43084001 [arXiv:1601.07459] (2016)

4. ROOT - An Object Oriented Data Analysis Framework, Rene Brun and Fons Rademakers, Nucl. Inst. \& Meth. in Phys. Res. A 389 (1997) 